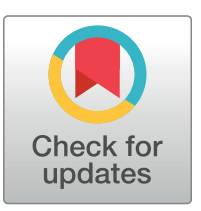

OPEn ACCESS

Citation: Jung YS, Han M, Kim DY, Cheon JH, Park $S$ (2017) Cancer risk in Korean patients with Behçet's disease: A nationwide population-based study. PLoS ONE 12(12): e0190182. https://doi. org/10.1371/journal.pone.0190182

Editor: James T. Rosenbaum, Oregon Health and Science University, UNITED STATES

Received: September 1, 2017

Accepted: December 8, 2017

Published: December 29, 2017

Copyright: @ 2017 Jung et al. This is an open access article distributed under the terms of the Creative Commons Attribution License, which permits unrestricted use, distribution, and reproduction in any medium, provided the original author and source are credited.

Data Availability Statement: All relevant data are within the paper.

Funding: This research was supported by two grants (A120176, HI13C1345) from the Korean Health Technology R\&D Project through the Korea Health Industry Development Institute (KHIDI) to $\mathrm{JHC}$, which is funded by the Ministry of Health and Welfare, Republic of Korea; and two grants (NRF2013R1A2A2A01067123, NRF-

2014R1A1A1008096) from the Basic Science

Research Program through the National Research Foundation of Korea, which is funded by the
RESEARCH ARTICLE

\section{Cancer risk in Korean patients with Behçet's disease: A nationwide population-based study}

\author{
Yoon Suk Jung ${ }^{1 \oplus}$, Minkyung Han ${ }^{2 \Phi}$, Do Young Kim³ , Jae Hee Cheon ${ }^{4 *}$, Sohee Park ${ }^{5 *}$ \\ 1 Division of Gastroenterology, Department of Internal Medicine, Kangbuk Samsung Hospital, \\ Sungkyunkwan University School of Medicine, Seoul, Republic of Korea, 2 Department of Public Health, \\ Graduate School, Yonsei University, Seoul, Republic of Korea, 3 Department of Dermatology and Cutaneous \\ Biology Research Institute, Yonsei University College of Medicine, Seoul, Republic of Korea, 4 Department of \\ Internal Medicine and Institute of Gastroenterology, Yonsei University College of Medicine, Seoul, Republic of \\ Korea, 5 Department of Biostatistics, Graduate School of Public Health, Yonsei University, Seoul, Republic of \\ Korea \\ ๑ These authors contributed equally to this work. \\ * soheepark@yuhs.ac (SP); GENIUSHEE@yuhs.ac (JHC)
}

\section{Abstract}

\section{Background}

Various immune-mediated diseases are associated with increased malignancy risks. However, the relationship between Behçet's disease (BD) and cancer remains unclear. We conducted a nationwide, population-based study to determine the risk of cancer in patients with BD.

\section{Methods}

Using National Health Insurance claims records, we collected data from 2402 patients diagnosed with BD between 2013 and 2014. Standardized incidence ratios (SIRs) of overall and site-specific cancers in patients with $\mathrm{BD}$ in comparison with the general population were calculated.

\section{Results}

The risks of overall cancer (SIR, 3.54; 95\% confidence interval, 2.35-5.11 in men and 2.17; 1.58-2.92 in women) and solid cancer (3.10; 1.94-4.69 in men and 2.13; 1.52-2.90 in women) were greater in patients with BD than in the general population. There were significantly increased risks for these solid cancers: colorectal $(4.26$; 1.38-9.94), liver $(4.00 ; 1.09$ $10.25)$, bone/articular cartilage (55.66; 1.41-310.14), prostate $(7.05 ; 1.45-20.60)$, and brain/central nervous system (28.32; 3.43-102.31) in men; and the lips/oral cavity/pharynx (13.97, 1.69-50.47), liver (12.78; 5.14-26.33), lungs (4.35; 1.18-11.13), other female genital organs $(53.57 ; 1.36-298.49)$, and eyes (128.26; 3.24-714.59) in women. Patients with $\mathrm{BD}$ had a greater risk of myelodysplastic syndrome (MDS) than the general population did (65.72; 7.96-237.41 in men and 53.86; 11.11-157.40 in women), but not of hematological cancer. 
Ministry of Science, ICT and Future Planning to $\mathrm{JHC}$. The funders had no role in study design, data collection and analysis, decision to publish, or preparation of the manuscript.

Competing interests: The authors have declared that no competing interests exist.

\section{Conclusions}

Compared to the general population, Korean patients with BD had greater risks of overall cancer, some solid cancers, and MDS, but not of hematological cancer.

\section{Introduction}

Behçet's disease (BD) is a chronic, multisystemic, immune-mediated disorder that is characterized by recurrent oral and/or genital ulcers, arthritis, and skin manifestations, as well as ocular, vascular, neurological, or intestinal involvement [1,2]. Autoimmunity and chronic inflammation are associated with malignancy development [3-5]. Immune-mediated diseases, which result from a dysregulated immune response, often cause chronic inflammation [3,4]. Chronic and unregulated inflammation can cause malignant cell transformation and carcinogenesis through inflammation-related mechanisms [3,4]. Chronic exposure of inflammatory mediators, including metabolites of arachidonic acids, cytokines, chemokines, and free radicals, leads to increased cell proliferation, mutagenesis, oncogene activation, and angiogenesis [3,4].

Several epidemiological studies have evaluated the relationships between a variety of systemic autoimmune rheumatic diseases and cancer, and the studies have demonstrated that rheumatoid arthritis (RA), systemic lupus erythematosus (SLE), Sjögren's syndrome, systemic sclerosis, and dermatomyositis, are associated with an increased risk of malignancy, especially lymphoma [5-9]. BD has also been reported to be sporadically associated with malignancy $[10,11]$. In particular, BD has been reported to be associated with hematological cancers in addition to bone marrow failure, such as myelodysplastic syndrome (MDS) and aplastic anemia (AA) [10-13]. Given that organs commonly involved in BD include the oral cavity, genitalia, skin, and eye and that $\mathrm{BD}$ recurrently induces chronic inflammation of these organs, $\mathrm{BD}$ may also confer a high risk of cancer in these organs. However, data on the relationship between $\mathrm{BD}$ and solid cancers are very scarce. Moreover, most previous studies investigating the associations between $\mathrm{BD}$ and cancer were case reports or hospital-based chart reviews with inconsistent results $[10,11,14]$. Thus, based on current evidence, it is difficult to conclude that $\mathrm{BD}$ itself increases the risk of cancer development.

To clarify the relationship between $\mathrm{BD}$ and cancer risk, we conducted a nationwide, population-based study to compare the overall and site-specific cancer risks in patients with BD against those in the general population using national insurance claims data in Korea.

\section{Material and methods}

\section{Data source}

As the Korean government operates a mandatory nationwide insurance system (National Health Insurance; NHI), all health care utilization information is registered under a comprehensive database operated by the Health Insurance and Review Agency (HIRA). Using the HIRA database allowed us to provide estimates based on the occurrence of events in unselected patient populations representing the whole spectrum of disease extent and severity.

This study used data from the NHI, which provides mandatory universal health insurance that covers all forms of health services, including hospitalization, ambulatory care, and pharmaceutical services. Medical institutions submit health care utilization information in electronic format for reimbursement purposes, and this information is integrated into the HIRA claims database, which covers the entire population of Korea (about 51 million people). The 
database contains information on all patients, including demographic characteristics, ambulatory care history, principal diagnosis and comorbidities using the International Classification of Diseases, 10th revision (ICD-10), prescriptions, and procedures.

Beginning in 2006, the NHI initiated a registration program for 138 rare intractable diseases (RIDs), including BD, in order to subsidize the medical expenses of patients with RIDs. Through the RID program, the Korean government covers $90 \%$ of the hospital expenses for patients with RIDs so that those patients pay for only $10 \%$ of their hospital expenses. Considering these medical benefits, a diagnosis of $\mathrm{BD}$ is not likely to be missed in the RID system. Additionally, the diagnosis must be made based on very strict diagnostic criteria provided by the RID system and must be reviewed by the corresponding healthcare institution before being submitted to the NHI. Thus, data regarding RIDs are verified and reliable. Our study only included cases of BD certified as RIDs in the HIRA claims database.

\section{Patient identification}

Patients identified as having BD in HIRA from January 2011 to December 2015 were included in this study. In the RID program, BD was diagnosed using the International Study Group diagnostic criteria for $\mathrm{BD}$ (recurrent oral ulcerations plus two of the following criteria: recurrent genital ulcerations, eye lesions, skin lesions, and positive pathergy test) [2]. To increase the diagnostic accuracy of BD, BD was determined in cases that met both the appropriate diagnostic code (ICD-10 code: M35.2) and the RID code (V139). The date of initial registration in the RID program during the study period was regarded as the date of $\mathrm{BD}$ diagnosis.

\section{Ascertainment of cancer incidence rates}

Cancer occurrence in $\mathrm{BD}$ patients was ascertained using the NHI database. For the comparison with the cancer incidence rates in the general population, we used the national database of The Korea Central Cancer Registry (KCCR) that covers the entire population in Korea [15]. Besides all incident cases of cancer (ICD-10: C00-C96), we also investigated the number of incident cases of MDS (ICD-10: D46) and AA (ICD-10: D61) because several previous studies have reported that $\mathrm{BD}$ is associated with these hematological diseases [10-12].

\section{Statistical analysis}

The source population for this study consisted of all patients with NHI claims data between 2011 and 2015. Because the inclusion of previous prevalent cases may confound the incidence, we applied a washout period of 2 years by excluding the patients that had any claim record for $\mathrm{BD}$ during these 2 years in recognition of these cases as prevalent rather than incident cases. To ensure accurate diagnosis, an incident case for $\mathrm{BD}$ was defined as a patient registered for BD for 2 years in a row. Thus, we could assess the incidence of BD between 2013 and 2014. The incidence rate was defined as the number of incident cases in the corresponding year per 100,000 people using the mid-year population size (resident registration population on July 1 of each year). Age and sex-specific incidence rates were calculated by dividing the number of cases in age- and sex-stratified groups by the corresponding age- and sex-specific population and was expressed as cases per 100,000 people.

To determine whether patients with $\mathrm{BD}$ had a greater risk of developing cancer than did the general population, we calculated the standardized incidence ratio (SIR, a ratio of observed to expected cancers) of overall and site-specific cancers in patients with BD. The number of observed cancer cases used only newly diagnosed cancer cases. The KCCR provides information on the general population's incidence rate of cancer in 10-year age intervals, from age 0 to 99 years. Using the sex- and age-specific incidence rates of the general population from KCCR 
data, the observed and expected numbers of cases in BD patients were calculated by sex- and age-specific groups. The number of expected cancer cases was calculated by multiplying the age-specific cancer incidence rate of the general population from 2013 and the person-years of patients with BD. The $95 \%$ confidence intervals (CIs) for the SIRs were calculated using Poisson's distribution.

To investigate the association between medication and the risk of cancer incidence, a nested case control analysis was used, which would avoid the length bias produced by a regular case control analysis and would match exposure durations of cases and controls. We also matched the diagnosis dates of $\mathrm{BD}$ to avoid temporal bias. For each subject who developed cancer during the follow-up period, four cancer-free subjects were randomly selected and matched to each cancer case with the same sex, age and diagnosis date of $\mathrm{BD}$. We then examined whether the cases and controls used medications during the period beginning with the diagnosis of $\mathrm{BD}$ and ending with each case's respective cancer diagnosis date (for controls, their matching case's respective cancer diagnosis date). Taking into account the matched case control data structure, we used conditional logistic regression to estimate the OR and corresponding 95\% CIs. SAS Enterprise Guide [SAS Institute, Inc.] was used for all statistical analyses. P values less than 0.05 were considered statistically significant.

\section{Ethical considerations}

All identifiable personal information in medical records was de-identified to comply with the privacy rule of the Health Insurance Portability and Accountability Act. In addition, as the information in the HIRA database is encrypted, the database does not contain personal identifiers. This study protocol was approved by the Institutional Review Board of Severance Hospital, Yonsei University College of Medicine (IRB No.4-2016-1135).

\section{Results}

\section{Study population and incidence}

The baseline characteristics of the study population are presented in Table 1. Overall, 2402 patients with BD were newly diagnosed between 2013 and 2014. The mean age at diagnosis was $44.5 \pm 13.1$ years, and the male-to-female ratio was 0.54 . The proportion of patients diagnosed with BD was highest in the $40-49$ year age group (29.4\%). Among all incident cases, 2178 (90.7\%), 1,986 (82.7\%), 373 (15.5\%), 599 (24.9\%), 662 (27.6\%), and 30 (1.2\%) were prescribed corticosteroids, colchicine, sulfasalazine, 5-aminosalicylic acid (ASA), thiopurine, and tumor necrosis factor alpha (TNF- $\alpha$ ) inhibitors (infliximab or adalimumab) during the followup period. Of the 30 patient who received TNF- $\alpha$ inhibitors, 13 (0.5\%) were treated using infliximab, and $19(0.8 \%)$ were treated using adalimumab.

The incidence of BD by sex and age group is shown in Fig 1. The incidences of BD in 2013 and 2014 were 2.44 and 2.30 per 100,000 person-years, respectively, with an average annual incidence of 2.37 per 100,000 person-years. The average annual incidences of BD in men and women were 1.67 and 3.07 per 100,000 person-years, respectively. The incidence of BD was highest in the $40-44$ year age group (4.27 per 100,000 person-years) and steadily decreased thereafter.

\section{Cancer risk in patients with Behçet's disease}

After excluding 89 patients with previous cancer, a total of 2313 patients with BD were included in the cancer SIR analysis and followed-up until December 31, 2015. The 89 excluded patients with previous cancers had 6 hematological cancers and 83 solid cancers (Fig 2). The 
Table 1. Baseline characteristics of the study population.

\begin{tabular}{l|c}
\hline Characteristic & $\mathbf{N}(\%)$ \\
\hline Total population & 2402 \\
\hline Men & $847(35.3)$ \\
\hline Women & $1555(64.7)$ \\
\hline Age at diagnosis (years; mean \pm SD) & $44.5 \pm 13.1$ \\
\hline$<20$ years & $72(3.0)$ \\
\hline $20-29$ years & $246(10.2)$ \\
\hline $30-39$ years & $500(20.8)$ \\
\hline $40-49$ years & $705(29.4)$ \\
\hline $50-59$ years & $614(25.6)$ \\
\hline $60-69$ years & $198(8.2)$ \\
\hline$\geq 70$ years & $67(2.8)$ \\
\hline Medication use & \\
\hline Corticosteroid & $2,178(90.7)$ \\
\hline Colchicine & $1,986(82.7)$ \\
\hline Sulfasalazine & $373(15.5)$ \\
\hline $5-A S A$ & $599(24.9)$ \\
\hline Thiopurines & $662(27.6)$ \\
\hline TNF- a inhibitors & $30(1.2)$ \\
\hline Infliximab & $13(0.5)$ \\
\hline Adalimumab & $19(0.8)$ \\
\hline
\end{tabular}

SD, standard deviation; 5-ASA, 5-aminosalicylic acid; TNF- $\alpha$, tumor necrosis factor alpha

https://doi.org/10.1371/journal.pone.0190182.t001

2313 patients with $\mathrm{BD}$ were followed for 5854 person-years (median follow-up duration, 2.34 years; range, $0.12-4.99$ years).

The SIRs of overall and site-specific cancers in patients with $\mathrm{BD}$ are presented in Table 2. Among the 823 men with BD, 28 cancers were observed (vs. 7.91 expected), which resulted in an increased overall cancer risk compared to that in the general population (SIR, 3.54; 95\% CI, 2.35-5.11). Compared with those in the general population, men with BD had significantly increased risks of colorectal cancer (CRC) (SIR, 4.26; 95\% CI, 1.38-9.94), liver cancer (SIR, 4.00; 95\% CI, 1.09-10.25), bone/articular cartilage cancer (SIR, 55.66; 95\% CI, 1.41-310.14), prostate cancer (SIR, 7.05; 95\% CI, 1.45-20.60), and brain/central nervous system (CNS) cancer (SIR, 28.32; 95\% CI, 3.43-102.31). Overall, 22 solid cancers were observed (vs. 7.11 expected), resulting in an increased overall solid cancer risk (SIR 3.10; 95\% CI 1.94-4.69). On the other hand, the risk of hematological cancer was not significantly higher in men with BD (SIR, 2.58; 95\% CI, 0.07-14.35); only one man with BD developed multiple myeloma vs. 0.04 expected (SIR, 22.44; 95\% CI, 0.57-125.05).

Among the 1490 women with BD, 44 cancers were observed (vs. 20.24 expected), which resulted in an increased overall cancer risk compared to that in the general population (SIR, 2.17; 95\% CI, 1.58-2.92). Similar to men with BD, women with BD had a significantly increased risk of overall solid cancer, but not of hematological cancer. Women with BD had increased risks of lip/oral cavity/pharynx cancer (SIR, 13.97; 95\% CI, 1.69-50.47), liver cancer (SIR, 12.78; 95\% CI, 5.14-26.33), lung cancer (SIR, 4.35; 95\% CI, 1.18-11.13), other female genital organ cancer (SIR, 53.57; 95\% CI, 1.36-298.49), and eye cancer (SIR, 128.26; 95\% CI, 3.24-714.59). Overall, 40 solid cancers were observed (vs. 18.79 expected), resulting in an increased overall solid cancer risk (SIR, 2.13; 95\% CI 1.52-2.90). However, none of women with $\mathrm{BD}$ developed hematological cancer. 


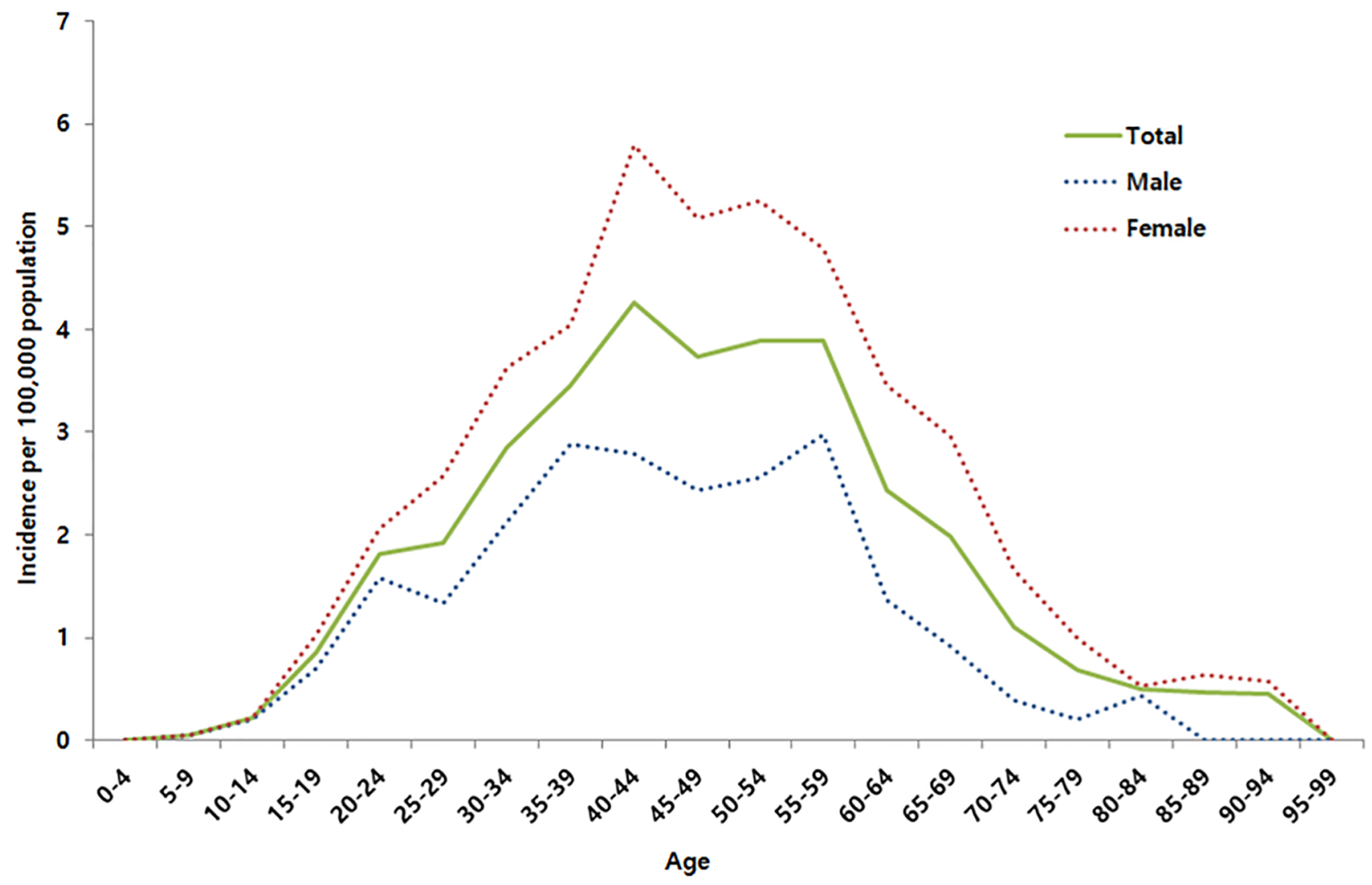

Fig 1. Incidence of Behçet's disease by sex and age group from 2013 to 2014.

https://doi.org/10.1371/journal.pone.0190182.g001

Because several studies have reported that BD is associated with MDS and AA [10-12], we further investigated the risk of these diseases in patients with $\mathrm{BD}$ in the present study. Two men and three women developed MDS; it followed that patients with BD had a significantly greater risk of MDS than the general population did (vs. 0.03 expected; SIR, 65.72; 95\% CI, 7.96-237.41 in men and vs. 0.06 expected; SIR, 53.86; 95\% CI, 11.11-157.40 in women). The SIR of AA in patients with $\mathrm{BD}$ was not calculated since the incidence rate of AA in the general population was not available; nonetheless, we identified six patients (two men and four women) who developed AA.

\section{Risk of cancer according to medication use}

The risk of solid cancer and overall cancer was lower in ever users of thiopurines than in never users of this drug (OR, 0.28; 95\% CI, 0.10-0.77 and 0.35; 0.14-0.86, respectively). However, the use of sulfasalazine or 5-ASA was not associated with the risk of solid cancer (OR, 1.51; 95\% CI, 0.72-3.15) or overall cancer (OR, 1.81; 95\% CI, 0.93-3.53) (Table 3). Because none with solid cancer or overall cancer used TNF- $\alpha$ inhibitors, we could not assess their association. Also, because only one man with BD developed hematological cancer, we could not assess the risk of hematological cancer according to medication use.

\section{Discussion}

In this first Korean population-based analysis of cancer risk in patients with BD, we found that patients (both men and women) with BD had greater risks of overall cancer and solid cancer 


\section{1 million people in Korean $\mathrm{NHI}$ database}

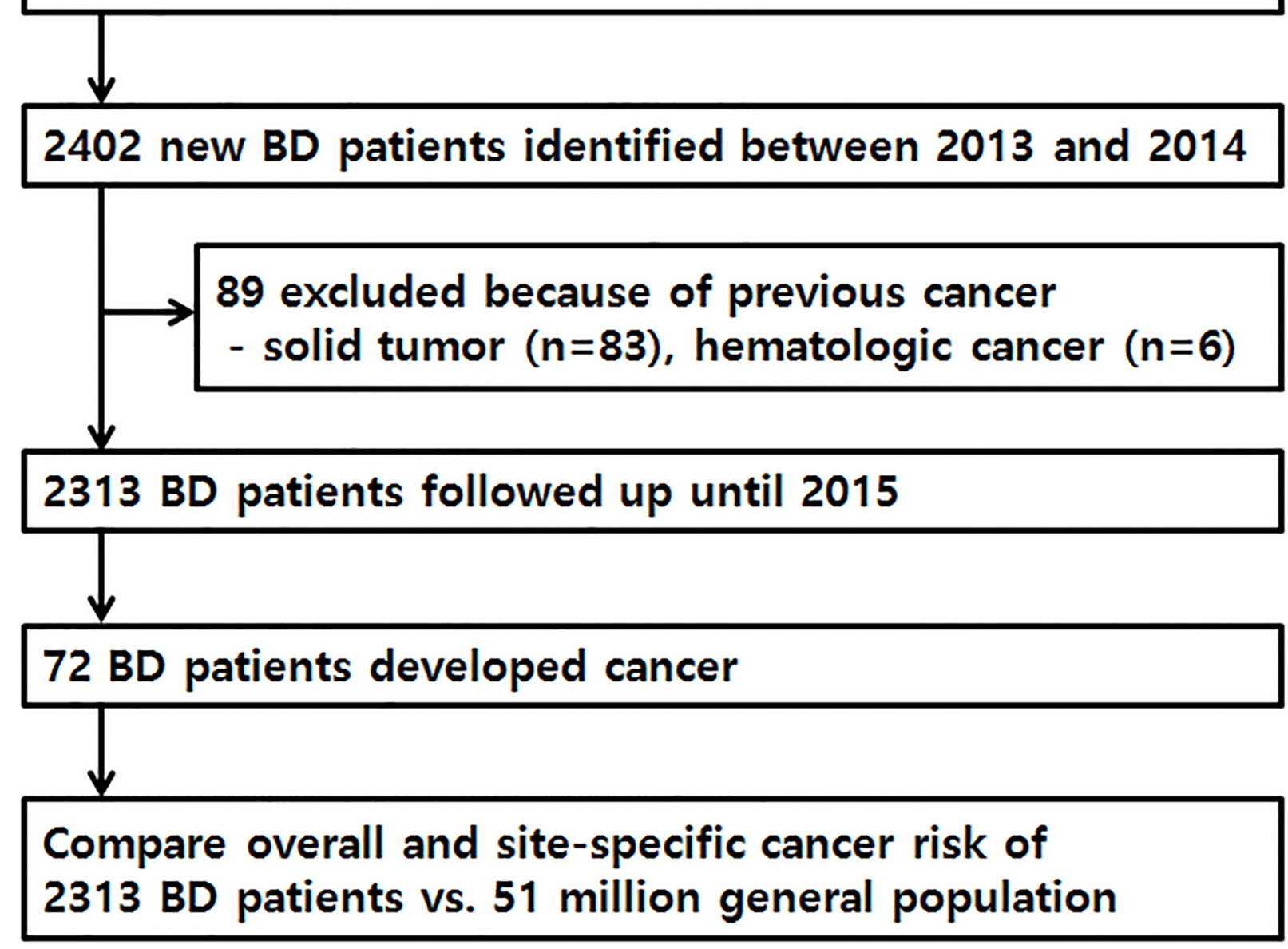

Fig 2. Flow chart of the study process.

https://doi.org/10.1371/journal.pone.0190182.g002

compared to those in the general population. Men with BD had an increased risk of CRC, liver cancer, bone/articular cartilage cancer, prostate cancer, and brain/CNS cancer. Women with $\mathrm{BD}$ had an increased risk of lip/oral cavity/pharynx cancer, liver cancer, lung cancer, other female genital organ cancer, and eye cancer. Patients (both men and women) with $\mathrm{BD}$ also had a greater risk of MDS than did the general population. However, the risk of hematological cancer in patients with $\mathrm{BD}$ was not greater than that in the general population.

To date, few population-based studies have been conducted to determine the cancer risk among patients with $\mathrm{BD}$. Only two recent studies have investigated the cancer risk in patients with BD at the population level in Taiwan $[16,17]$. Similar to our results, the Taiwan study of 1314 new BD patients identified between 2000 and 2009 demonstrated that patients with BD had a greater risk of overall cancer (SIR 1.5, 95\% CI 1.03-2.1) [16].

Given that $\mathrm{BD}$ is a chronic, multisystemic, immune-mediated disorder that is characterized by oral, genital, skin, ocular, intestinal, and/or neurological involvement, it is interesting, but not surprising, that we found that patients with $\mathrm{BD}$ had a greater risk of solid cancer than did the general population. The increased risk in patients with $\mathrm{BD}$ was particularly evident for solid cancers in organs that can be involved in $\mathrm{BD}$ (such as the colon and rectum, articular cartilage, and brain/CNS in men and the lips/oral cavity/pharynx, female genital organ, and eyes 
Table 2. Risks of site-specific cancer in patients with Behçet's disease.

\begin{tabular}{|c|c|c|c|c|c|c|}
\hline \multirow[t]{2}{*}{ Cancer (ICD-10 code) } & \multicolumn{3}{|c|}{ Men } & \multicolumn{3}{|c|}{ Women } \\
\hline & Observed, $n$ & Expected, $\mathrm{n}$ & $\operatorname{SIR}(95 \% \mathrm{Cl})$ & Observed, $n$ & Expected, $\mathbf{n}$ & SIR (95\%Cl) \\
\hline All cancer (C00-C96) & 28 & 7.91 & $3.54(2.35-5.11)$ & 44 & 20.24 & $2.17(1.58-2.92)$ \\
\hline Solid cancer & 22 & 7.11 & $3.10(1.94-4.69)$ & 40 & 18.79 & $2.13(1.52-2.90)$ \\
\hline Lip, oral cavity, and pharynx (C00-C14) & - & & & 2 & 0.14 & $13.97(1.69-50.47)$ \\
\hline Stomach $(\mathrm{C} 16)$ & 3 & 1.48 & $2.03(0.42-5.93)$ & 4 & 1.50 & $2.67(0.73-6.82)$ \\
\hline Colon and rectum (C18-C20) & 5 & 1.17 & $4.26(1.38-9.94)$ & 2 & 1.61 & $1.24(0.15-4.49)$ \\
\hline Liver (C22) & 4 & 1.00 & $4.00(1.09-10.25)$ & 7 & 0.55 & $12.78(5.14-26.33)$ \\
\hline Gallbladder (C23-C24) & - & & & 1 & 0.27 & $3.69(0.09-20.54)$ \\
\hline Pancreas (C25) & 1 & 0.19 & $5.39(0.14-30.03)$ & 1 & 0.29 & $3.40(0.09-18.94)$ \\
\hline Lung (C33-C34) & 1 & 0.82 & $1.22(0.03-6.81)$ & 4 & 0.92 & $4.35(1.18-11.13)$ \\
\hline Bone and articular cartilage (C40-C41) & 1 & 0.02 & $55.66(1.41-310.14)$ & - & & \\
\hline Skin (C43-C44) & 1 & 0.13 & $7.95(0.20-44.29)$ & 1 & 0.28 & $3.55(0.09-19.78)$ \\
\hline Breast (C50) & - & & & 3 & 3.90 & $0.77(0.16-2.25)$ \\
\hline Cervix, uterus (C53) & - & & & 2 & 0.71 & $2.80(0.34-10.12)$ \\
\hline Other female genital organ (C57) & - & & & 1 & 0.02 & $53.57(1.36-298.49)$ \\
\hline Prostate (C61) & 3 & 0.43 & $7.05(1.45-20.60)$ & - & & \\
\hline Kidney (C64) & - & & & 1 & 0.23 & $4.41(0.11-24.55)$ \\
\hline Eye (C69) & - & & & 1 & 0.01 & $128.26(3.24-714.59)$ \\
\hline Brain and CNS (C70-C72) & 2 & 0.07 & $28.32(3.43-102.31)$ & 1 & 0.13 & $7.50(0.19-41.80)$ \\
\hline Thyroid (C73) & 1 & 0.95 & $1.05(0.03-5.86)$ & 9 & 7.45 & $1.21(0.55-2.29)$ \\
\hline Hematological cancer & 1 & 0.39 & $2.58(0.07-14.35)$ & & & \\
\hline Multiple myeloma (C90) & 1 & 0.04 & $22.44(0.57-125.05)$ & - & & \\
\hline Other (remaining cancer codes) & 5 & 0.42 & $11.89(3.86-27.75)$ & 4 & 0.82 & $4.89(1.33-12.52)$ \\
\hline
\end{tabular}

ICD-10, International Classification of Diseases, $10^{\text {th }}$ revision; SIR, standardized incidence ratio; $\mathrm{Cl}$, confidence interval, CNS, central nervous system. Bold text in this table indicates statistically significant results.

in women). A dysregulated immune response can cause chronic inflammation, which may in turn, through inflammatory mediators, cause malignant cell transformation and carcinogenesis in involved organs [3,4]. Several studies have reported a relationship between BD and hematologic cancers or diseases [10-13], whereas research on the association of BD with the risk of solid cancer has been rare. A Chinese study involving $41 \mathrm{BD}$ patients who developed malignancies demonstrated that CRC was the most common solid tumor associated with $\mathrm{BD}$ [10]. A Korean study reported that of 1769 patients with BD, 21 (1.2\%) developed solid cancer,

Table 3. Risk of cancer according to medication use in patients with Behçet's disease.

\begin{tabular}{|c|c|c|}
\hline & Solid cancer OR & Overall cancer OR \\
\hline \multicolumn{3}{|c|}{ Sulfasalazine or 5-ASA ever use } \\
\hline No & 1 (Reference) & 1 (Reference) \\
\hline Yes & $1.51(0.72-3.15)$ & $1.81(0.93-3.53)$ \\
\hline \multicolumn{3}{|c|}{ Thiopurines ever use } \\
\hline No & 1 (Reference) & 1 (Reference) \\
\hline Yes & $0.28(0.10-0.77)$ & $0.35(0.14-0.86)$ \\
\hline
\end{tabular}

5-ASA, 5-aminosalicylic acid; OR, odds ratio

Bold text in this table indicates statistically significant results.

https://doi.org/10.1371/journal.pone.0190182.t003 
and thyroid cancer was the most common solid cancer occurring in patients with $\mathrm{BD}$ [11]. However, these two studies simply presented descriptive statistics for the most common solid cancers among patients with $\mathrm{BD}$; thus, based on their results, it was difficult to determine whether patients with BD had a greater risk of those solid cancers than did people in the general population. To date, only one study has reported an increased risk of certain solid cancers in patients with $\mathrm{BD}$ at the population level. A Taiwan population-based study revealed that women with $\mathrm{BD}$ had a greater risk of breast cancer than did the general population (SIR 2.2, 95\% CI 1.004-4.1) [16]. Our study is the first to show that the risk of some solid cancers, especially cancers of organs that can be involved in $\mathrm{BD}$, is higher in patients with $\mathrm{BD}$ than that in the general population.

Contrary to the results for solid cancer, the risk of hematologic cancers was not greater in patients with BD than that in the general population. Many previous studies have reported greater risks of hematological cancers in patients with autoimmune diseases such as RA, SLE, Sjögren's syndrome, systemic sclerosis, and dermatomyositis [5-9]. Recently, two Taiwanese population-based studies also demonstrated that patients with $\mathrm{BD}$ had a greater risk of hematological malignancies (SIR, 4.2), especially non-Hodgkin's lymphoma (SIR, 6.2-8.3) [16,17]. Immune-mediated diseases and hematological cancers have similar genetic susceptibilities and environmental triggers (e.g., Epstein-Barr virus infection) [18], and they also share a common pathogenesis. Several inflammatory cytokines, including tumor necrosis factor- $\alpha$ (TNF- $\alpha$ ), interferon- $\gamma$, interleukin (IL)- 6 , and IL-8, play a role in the pathogenesis of both immunemediated diseases and hematologic cancers [19-22]. These shared factors are thought to be involved in the development of hematologic cancers in patients with immune-mediated diseases. However, contrary to our expectation, only one of 2313 patients with $\mathrm{BD}$ in the present study developed hematological cancer (multiple myeloma), and BD was not significantly associated with a greater risk of hematologic cancer. The main reason for this unexpected result may be that the follow-up period in the present study was too short (median, 2.34 years). In the future, long-term studies are needed to clarify the causal relationship between $\mathrm{BD}$ and hematological cancer.

However, based on our results, it is difficult to conclude that $\mathrm{BD}$ does not increase the risk of hematological cancer because the risk of MDS, which can progress to acute myeloid leukemia [23], was significantly greater in patients with $\mathrm{BD}$ than that in the general population. Moreover, the risk of MDS was much greater in comparison to that of other significant solid cancers (SIR, 65.7 in men and SIR, 53.9 in women). Several previous studies have also reported that BD is associated with MDS [10-13]. Furthermore, a review of the literature reported that MDS is markedly more common than leukemia is in Japanese BD patients [12]. Cytogenetic aberrations, especially trisomy 8 , are thought to play an important role in the pathogenesis of $\mathrm{BD}$ associated with MDS. Of the cytogenetic abnormalities, trisomy 8 has been reported to be most common, occurring in $64-87 \%$ of patients with BD associated with MDS [12,13]. However, all of the previous studies regarding BD associated with MDS were case reports or hospital-based studies. Our study is meaningful in that it is the first population-based study to show that $\mathrm{BD}$ increases the risk of MDS.

In the present study, the use of thiopurines was associated with lower solid and overall cancer risk in patients with BD. Our results may be in accord with findings of studies of patients with inflammatory bowel disease (IBD). Some studies have shown that immunomodulators reduce the risk of colorectal cancer by suppressing intestinal inflammation in patients with IBD $[24,25]$. Similar to patients with IBD, immunomodulators may reduce the risk of solid cancer by suppressing the inflammation of the affected organ in patients with BD. Several other studies have reported that immunomodulators may increase the risk of hematological cancers [26]. However, we could not assess the relationship between the use of thiopurines and 
the risk of hematological cancers because only one patient developed hematological cancer. In our study, the follow-up duration was too short to thoroughly evaluate the effect of medication use on risk of cancer. Future extended, long-term studies are required to elucidate the effects of use of medication including thiopurines and TNF- $\alpha$ inhibitors on cancer development.

Our study had several limitations. First, we did not verify the diagnostic accuracy of BD. As we used insurance claims data, it is possible that some patients with BD were missed or that patients without $\mathrm{BD}$ had been misdiagnosed. To overcome this limitation, we only included cases that met the RID code as well as the BD diagnostic code. Given that patients can be registered in the RID system only if they meet strict diagnostic criteria, BD is not likely to be misdiagnosed. In addition, given the medical benefits provided by the RID program, a diagnosis of $\mathrm{BD}$ is not likely to be missed. Therefore, we believe that the definition of $\mathrm{BD}$ diagnosis in the present study was reliable. Second, since we used administrative data, we could not obtain information on symptoms or signs of $\mathrm{BD}$ and the organs that were involved. For example, patients with intestinal involvement may have a greater risk of CRC and patients with neurological involvement may have a greater risk of brain/CNS cancer. However, we could not evaluate the cancer risk according to the involved organs. Third, the duration of follow-up was too short to assess completely the development of BD-associated cancers. We could evaluate cancer risk in the early stages of the disease, but not the longterm cancer risk. Nevertheless, our study showed that patients with BD had an increased risk of overall cancer, solid cancers, and MDS in the early stages of the disease. Many patients with $\mathrm{BD}$ may have symptoms of $\mathrm{BD}$, such as recurrent oral ulcers, before an objective diagnosis is made. Additionally, patients with a predisposition toward developing BD may have dysregulated immune responses even before the diagnosis of $\mathrm{BD}$ is made. These may result in a high risk of cancer even in the early stage of the disease. Finally, there might have been a detection bias. Patients with BD are more likely to seek health care and to need multiple hospital visits, as compared to the general population, and thus they may have a greater rate of cancer detection than the general population does.

In conclusion, both men and women with $\mathrm{BD}$ had a greater risk of overall cancer, some solid cancers (especially in organs that can be involved in BD), and MDS than the general population did. However, the risk of hematological cancer in patients with BD was not significantly greater than that in the general population. Further long-term cohort studies are required to clarify the influence of $\mathrm{BD}$ itself, and medications used for its treatment, on cancer development.

\section{Acknowledgments}

This research was supported by two grants (A120176, HI13C1345) from the Korean Health Technology R\&D Project through the Korea Health Industry Development Institute (KHIDI), which is funded by the Ministry of Health and Welfare, Republic of Korea; and two grants (NRF-2013R1A2A2A01067123, NRF-2014R1A1A1008096) from the Basic Science Research Program through the National Research Foundation of Korea, which is funded by the Ministry of Science, ICT and Future Planning.

\section{Author Contributions}

Conceptualization: Yoon Suk Jung, Minkyung Han, Do Young Kim, Jae Hee Cheon, Sohee Park.

Data curation: Yoon Suk Jung, Minkyung Han, Jae Hee Cheon, Sohee Park.

Formal analysis: Yoon Suk Jung, Minkyung Han, Sohee Park. 
Funding acquisition: Jae Hee Cheon.

Investigation: Yoon Suk Jung, Minkyung Han, Do Young Kim, Jae Hee Cheon, Sohee Park.

Methodology: Yoon Suk Jung, Minkyung Han, Jae Hee Cheon, Sohee Park.

Project administration: Yoon Suk Jung, Minkyung Han.

Resources: Yoon Suk Jung, Minkyung Han.

Supervision: Yoon Suk Jung, Minkyung Han, Do Young Kim, Jae Hee Cheon, Sohee Park.

Validation: Yoon Suk Jung, Minkyung Han, Sohee Park.

Visualization: Yoon Suk Jung, Minkyung Han, Sohee Park.

Writing - original draft: Yoon Suk Jung, Minkyung Han.

Writing - review \& editing: Minkyung Han, Do Young Kim, Sohee Park.

\section{References}

1. Cheon JH, Kim WH. An update on the diagnosis, treatment, and prognosis of intestinal Behçet's disease. Curr Opin Rheumatol. 2015; 27: 24-31. https://doi.org/10.1097/BOR.0000000000000125 PMID: 25405821

2. Dalvi SR, Yildirim R, Yazici Y. Behcet's Syndrome. Drugs. 2012; 72: 2223-2241. https://doi.org/10. 2165/11641370-000000000-00000 PMID: 23153327

3. Beyaert R, Beaugerie L, Van Assche G, Brochez L, Renauld JC, Viguier M, et al. Cancer risk in immune-mediated inflammatory diseases (IMID). Mol Cancer. 2013; 12: 98. https://doi.org/10.1186/ 1476-4598-12-98 PMID: 23987103

4. Okada F. Inflammation-related carcinogenesis: current findings in epidemiological trends, causes and mechanisms. Yonago Acta Med. 2014; 57: 65-72. PMID: 25324587

5. Turesson C, Matteson EL. Malignancy as a comorbidity in rheumatic diseases. Rheumatology (Oxford). 2013; 52: 5-14.

6. Weng MY, Huang YT, Liu MF, Lu TH. Incidence of cancer in a nationwide population cohort of 7852 patients with primary Sjogren's syndrome in Taiwan. Ann Rheum Dis. 2012; 71: 524-527. https://doi. org/10.1136/annrheumdis-2011-200402 PMID: 22072014

7. Chen YJ, Chang YT, Wang CB, Wu CY. The risk of cancer in patients with rheumatoid arthritis: a nationwide cohort study in Taiwan. Arthritis Rheum. 2011; 63: 352-358. https://doi.org/10.1002/art.30134 PMID: 21279991

8. Liang JA, Sun LM, Yeh JJ, Lin WY, Chang SN, Sung HC, et al. Malignancies associated with systemic lupus erythematosus in Taiwan: a nationwide population-based cohort study. Rheumatol Int. 2012; 32: 773-778. https://doi.org/10.1007/s00296-010-1684-y PMID: 21193991

9. Kuo CF, See LC, Yu KH, Chou IJ, Chang HC, Chiou MJ, et al. Incidence, cancer risk and mortality of dermatomyositis and polymyositis in Taiwan: a nationwide population study. Br J Dermatol. 2011; 165: 1273-1279. https://doi.org/10.1111/j.1365-2133.2011.10595.x PMID: 21895620

10. Lin Y, Li G, Zheng W, Tian X, Zhang F. Behcet's disease associated with malignancy: a report of 41 Chinese cases. Int J Rheum Dis. 2014; 17: 459-465. https://doi.org/10.1111/1756-185X.12269 PMID: 24354961

11. Ahn JK, Oh JM, Lee J, Koh EM, Cha HS. Behcet's disease associated with malignancy in Korea: a single center experience. Rheumatol Int. 2010; 30: 831-835. https://doi.org/10.1007/s00296-009-1319-3 PMID: 20016987

12. Tada $Y$, Koarada S, Haruta $Y$, Mitamura M, Ohta A, Nagasawa $K$. The association of Behcet's disease with myelodysplastic syndrome in Japan: a review of the literature. Clin Exp Rheumatol. 2006; 24: S115-119. PMID: 17067441

13. Ahn JK, Cha HS, Koh EM, Kim SH, Kim YG, Lee CK, et al. Behcet's disease associated with bone marrow failure in Korean patients: clinical characteristics and the association of intestinal ulceration and trisomy 8. Rheumatology (Oxford). 2008; 47: 1228-1230.

14. Na SY, Shin J, Lee ES. Morbidity of solid cancer in Behcet's disease: analysis of 11 cases in a series of 506 patients. Yonsei Med J. 2013; 54: 895-901. https://doi.org/10.3349/ymj.2013.54.4.895 PMID: 23709423 
15. Korea Central Cancer Registry, National Cancer Center. Annual report of cancer statistics in Korea in 2013, Ministry of Health and Welfare, 2015.

16. Wang LH, Wang WM, Hsu SM, Lin SH, Shieh CC. Risk of Overall and Site-specific Cancers in Behcet Disease: A Nationwide Population-based Study in Taiwan. J Rheumatol. 2015; 42: 879-884. https://doi. org/10.3899/jrheum.140770 PMID: 25834207

17. Yu KH, Kuo CF, Huang LH, Huang WK, See LC. Cancer Risk in Patients With Inflammatory Systemic Autoimmune Rheumatic Diseases: A Nationwide Population-Based Dynamic Cohort Study in Taiwan. Medicine (Baltimore). 2016; 95: e3540.

18. Baecklund E, Smedby KE, Sutton LA, Askling J, Rosenquist R. Lymphoma development in patients with autoimmune and inflammatory disorders-what are the driving forces? Semin Cancer Biol. 2014; 24: 61-70. https://doi.org/10.1016/j.semcancer.2013.12.001 PMID: 24333759

19. Hamzaoui K, Hamzaoui A, Guemira F, Bessioud M, Hamza M, Ayed K. Cytokine profile in Behcet's disease patients. Relationship with disease activity. Scand J Rheumatol. 2002; 31: 205-210. PMID: 12369651

20. Bardak $\mathrm{Y}$, Aridogan $\mathrm{BC}$. The demonstration of serum interleukin 6-8, tumor necrosis factor-alpha, complement, and immunoglobulin levels in Behcet's disease with ocular involvement. Ocul Immunol Inflamm. 2004; 12: 53-58. PMID: 15209464

21. Voulgarelis M, Giannouli S, Ritis K, Tzioufas AG. Myelodysplasia-associated autoimmunity: clinical and pathophysiologic concepts. Eur J Clin Invest. 2004; 34: 690-700. https://doi.org/10.1111/j.1365-2362. 2004.01417.x PMID: 15473894

22. Hsu HC, Lee YM, Tsai WH, Jiang ML, $\mathrm{Ho} \mathrm{CH}, \mathrm{Ho} C K$, et al. Circulating levels of thrombopoietic and inflammatory cytokines in patients with acute myeloblastic leukemia and myelodysplastic syndrome. Oncology. 2002; 63: 64-69. https://doi.org/10.1159/000065722 PMID: 12187073

23. Gangat $\mathrm{N}$, Patnaik MM, Tefferi A. Myelodysplastic syndromes: Contemporary review and how we treat. Am J Hematol. 2016; 91: 76-89. https://doi.org/10.1002/ajh.24253 PMID: 26769228

24. Andersen NN, Jess T. Has the risk of colorectal cancer in inflammatory bowel disease decreased? World J Gastroenterol. 2013; 19: 7561-7568. https://doi.org/10.3748/wjg.v19.i43.7561 PMID: 24282346

25. Jess T, Simonsen J, Jorgensen KT, Pedersen BV, Nielsen NM, Frisch M. Decreasing risk of colorectal cancer in patients with inflammatory bowel disease over 30 years. Gastroenterology. 2012; 143: 375381.e371; quiz e313-374. https://doi.org/10.1053/j.gastro.2012.04.016 PMID: 22522090

26. Kotlyar DS, Lewis JD, Beaugerie L, Tierney A, Brensinger CM, Gisbert JP, et al. Risk of lymphoma in patients with inflammatory bowel disease treated with azathioprine and 6-mercaptopurine: a meta-analysis. Clin Gastroenterol Hepatol. 2015; 13: 847-858.e844; quiz e848-850. https://doi.org/10.1016/j.cgh. 2014.05.015 PMID: 24879926 